SLAC-PUB-8280

SCIPP 99/45

October 1999

$(\mathrm{T} / \mathrm{E})$

\title{
Search for Charmless Hadronic Decays of $B$ Mesons with the SLD Detector
}

\author{
The SLD Collaboration ${ }^{\dagger}$ \\ Stanford Linear Accelerator Center \\ Stanford University, Stanford, CA 94309
}

\begin{abstract}
Based on a sample of approximately 500,000 hadronic $Z^{0}$ decays accumulated between 1993 and 1998, the SLD experiment has set limits on 24 fully charged two-body and quasi two-body exclusive charmless hadronic decays of $B^{+}, B^{0}$, and $B_{s}^{0}$ mesons. The precise tracking capabilities of the SLD detector provided for the efficient reduction of combinatoric backgrounds, yielding the most precise available limits for ten of these modes.
\end{abstract}

\author{
Submitted to Physical Review D - Rapid Communications
}

*Work supported by Department of Energy contract DE-AC03-76SF00515 (SLAC). 
The search for exclusive charmless decays of $B$ mesons is motivated by the CKMsuppression of the $W$-boson mediated $b \rightarrow u$ transition, which suppresses the leading order weak decay to charmless final states by a factor of $\left|V_{u b}\right|^{2} /\left|V_{c b}\right|^{2} \simeq 10^{-2}$ relative to that of charmed final states. Thus, observation of exclusive charmless modes with even modest branching fractions can indicate the participation of heretofore unobserved physical processes.

Recently, several results have increased the interest in exclusive charmless $B$ meson decays. The CLEO collaboration [1] has improved their measurement of the decay $B \rightarrow$ $\eta K^{*}$, with the measured branching fractions $B r\left(B^{+} \rightarrow \eta K^{*+}\right)=\left(2.73_{-0.82}^{+0.96} \pm 0.50\right) \times 10^{-5}$ and $\operatorname{Br}\left(B^{0} \rightarrow \eta K^{* 0}\right)=\left(1.38_{-0.44}^{+0.55} \pm 0.17\right) \times 10^{-5}$ somewhat above the expected range [2] of $(0.02-0.82) \times 10^{-5}$ and $(0.01-0.89) \times 10^{-5}$, respectively. In addition, the DELPHI collaboration has reported a measurement [3] of the combined mode $\operatorname{Br}\left(B^{+} \rightarrow \rho^{0} \pi^{+}+\right.$ $\left.K^{* 0} \pi^{+}\right)=\left(17_{-8}^{+12} \pm 2\right) \times 10^{-5}$, again somewhat higher than both the expected range [2] of $(0.4-2.0) \times 10^{-5}$ and the corresponding CLEO measurements 四 of $\operatorname{Br}\left(B^{+} \rightarrow \rho^{0} \pi^{+}\right)=$ $\left(1.5_{-0.5}^{+0.5} \pm 0.4\right) \times 10^{-5}$ and $\operatorname{Br}\left(B^{+} \rightarrow K^{* 0} \pi^{+}\right)<2.7 \times 10^{-5}$.

In this Letter, we present limits from the SLD detector on several two, three, and fourprong fully charged two-body and quasi-two-body final states. Although the $B$ meson sample available to the SLD detector is fairly limited in comparison to those produced at LEP, CESR, and the TEVATRON, the excellent tracking and a prior $i$ knowledge of the $B$ meson production point admit limits competitive with those produced elsewhere. Most limits presented here on four-prong final states, for which combinatoric backgrounds are worst, are the first available. In addition, the cms energy available to experiments running at the $Z^{0}$ pole allows the study of $B_{s}$ decays, which are inaccessible to experiments running at the $\Upsilon(4 S)$.

Search modes reported in this Letter include $B^{0}, B_{s}^{0} \rightarrow P^{+} P^{-}$(two-prong), $B^{+} \rightarrow P^{+} V^{0}$ (three-prong), and $B^{0}, B_{s}^{0} \rightarrow V^{0} V^{0}$ (four-prong), and their charge-conjugates, where $P=$ $\pi, K$ is a stable pseudoscalar meson and $V=\rho^{0}, K^{* 0}, \phi$ is a vector meson resonance with a sizeable branching fraction into two charged pseudoscalar mesons $(100 \%, 66.7 \%$, and $(49.1$ $\pm 0.8) \%$ [5], respectively). The ability to fully reconstruct the decaying $B$ meson state, with precise momentum and vertex information for each of the charged daughter tracks, provides an essential constraint in the analysis; no attempt was made to search for modes with one or more long-lived final state neutral particles.

The SLD detector [6] instruments the sole interaction region of the SLAC Linear Collider (SLC). The luminous region of the SLC is an ellipsoid of dimensions approximately 2 and $0.8 \mu \mathrm{m}$ in the horizontal $(x)$ and vertical (y) directions perpendicular to the beam axis, and $700 \mu \mathrm{m}$ along the beam axis. Due to motion of the collision point, however, the location of the luminous region is known to only $\sim 7 \mu m$ in $x$ and $y$.

Charged particle tracks are reconstructed in the central drift chamber (CDC) and the CCD-based pixel vertex detector (VXD) in a uniform axial magnetic field of 0.6T. Including the uncertainty in the location of the luminous region (IP) of the SLC, the VXD2 vertex detector, in place through 1995, exhibited an $r-\phi(r-z)$ impact parameter resolution of $11 \mu \mathrm{m}(38 \mu \mathrm{m})$ at high momentum, and $71 \mu \mathrm{m}(80 \mu \mathrm{m})$ at $p_{\perp} \sqrt{\sin \theta}=1.0 \mathrm{GeV} / \mathrm{c}$ [7]. The corresponding resolution for the VXD3 vertex detector [8], in place since 1996, is $14 \mu \mathrm{m}$ $(26.5 \mu m)$ at high momentum, and $33 \mu m(33 \mu m)$ at $p_{\perp} \sqrt{\sin \theta}=1.0 \mathrm{GeV} / \mathrm{c}$. The combined $\mathrm{CDC} / \mathrm{VXD}$ momentum resolution in the plane perpendicular to the beam axis is $\delta p_{\perp} / p_{\perp}=$ 
$\sqrt{(.01)^{2}+\left(.0026 p_{\perp} / \mathrm{GeV} / \mathrm{c}\right)^{2}}$. High momentum charged tracks are reconstructed in the range $|\cos \theta|<0.85$, with an efficiency of $96 \%$ for $|\cos \theta|<0.65$. A segmented Si-W forward calorimeter, with polar angle acceptance between $23 \mathrm{mr}$ and $68 \mathrm{mr}$, is used to monitor the SLC luminosity via t-channel Bhabha scattering.

The SLD accumulated an integrated luminosity of $19.1 \mathrm{pb}^{-1}$ of $e^{+} e^{-}$annihilation data at the $Z^{0}$ pole between 1993 and 1998. Of this, $14.0 p b^{-1}$ was taken with the upgraded VXD3 vertex detector in place.

The complete reconstruction of the fully-charged final state provides a number of constraints which can be used to discriminate between signal and potential background sources. Candidate track combinations must be consistent with having arisen from a single vertex. This vertex is displaced from the collision point by an average of $\sim 3 \mathrm{~mm}$, which is measured with an average uncertainty of $60 \mu \mathrm{m}$ for the search modes. The point of closest approach of the extrapolated vertex momentum resultant to the SLD IP ('vertex impact parameter') must be consistent with zero. The invariant mass of the tracks forming the vertex must be consistent with that of the $\mathrm{B}$ meson and have a total momentum consistent with known fragmentation properties. Tracks emerging from the $B$-meson decay vertex should have a relatively small opening angle, a large momentum, and a relatively large impact parameter with respect to the SLD IP. For quasi two-body modes involving vector meson resonances $(B \rightarrow P V$ and $B \rightarrow V V)$, two of the charged tracks must have an invariant mass consistent with that of each resonance. In addition, for $B \rightarrow P V$ modes, the decay angle $\theta_{h}$ of the $V$ state with respect to its flight direction ('helicity angle') must be consistent with the distribution $d \Gamma / d \Omega_{h} \propto \cos ^{2} \theta_{h}$ dictated by angular momentum conservation.

Candidate decays were reconstructed by considering all combinations of two, three, and four tracks which pass track quality cuts [6] and with total charge 0 for $P P$ and $V V$ candidates, and \pm 1 for $P V$ candidates. The invariant mass of the candidate decay was required to be above $5.05 \mathrm{GeV} / \mathrm{c}^{2}\left(5.15 \mathrm{GeV} / \mathrm{c}^{2}\right)$ for $B^{+}$and $B^{0}\left(B_{s}^{0}\right)$ modes. The probability of the vertex fit to the candidate tracks was required to be greater than $1.0 \%(0.5 \%$ for $B \rightarrow V V$ modes and $B \rightarrow P V$ with $V=\phi$ ), with a significance (separation from the SLD IP divided by the associated error) of greater than 1.0 (0.6 for $B \rightarrow V V$ modes and $B \rightarrow P V$ with $V=\phi)$. The smallest impact parameter $D$, normalized to its corresponding uncertainty, of any track in the candidate vertex was required to be greater than 1.1 (0.6 for $B \rightarrow V V$ modes and $B \rightarrow P V$ modes with $V=\phi)$. The change in the vertex invariant mass between the assumption of the nominal (kaon) mass and pion mass for all relevant tracks in the candidate vertex was required to be less than $0.3 \mathrm{GeV} / \mathrm{c}^{2}\left(1.2 \mathrm{GeV} / \mathrm{c}^{2}\right)$ for the $B \rightarrow P K^{ \pm}\left(B \rightarrow K \rho^{0}\right)$ modes; this cut suppresses background vertices which get an artificially large mass due to a mistaken mass hypothesis for one or more tracks. A second mass reconstruction quantity $\mathcal{M}_{V V}$, defined to be the sum of the absolute values of the differences between the reconstructed and nominal masses of the vector meson and $B$ meson candidates, was required to be less than $0.6 \mathrm{GeV} / \mathrm{c}^{2}, 0.4 \mathrm{GeV} / \mathrm{c}^{2}$, and $0.4 \mathrm{GeV} / \mathrm{c}^{2}$ for the $B \rightarrow \rho^{0} \rho^{0}, B \rightarrow \rho^{0} V\left(V \neq \rho^{0}\right)$, and $B_{s}^{0} \rightarrow \bar{K}^{* 0} K^{* 0}$ modes, respectively. For relevant modes, the reconstructed vector meson masses were required to be in the ranges $[0.2-1.1],[0.7-1.0]$, and $[1.000-1.035] \mathrm{GeV} / \mathrm{c}^{2}$ for $V=\rho^{0}, K^{* 0}$, and $\phi$. Finally, $\left|\cos \theta_{h}\right|$ was required to be greater than 0.3 for $B \rightarrow P V$ modes.

To further suppress background, an $a d-h o c$ discriminator function was devised, and tuned to a sample of Monte Carlo (MC) $Z^{0} \rightarrow b \bar{b}$ events approximately ten times that of data, and 
a sample of light quark (udsc) events approximately four times that of data. For $B \rightarrow P P$ modes, this function took the form

$$
\begin{array}{r}
\mathcal{F}_{P P}=a_{0} e^{-\frac{\left(m-M_{b}\right)^{2}}{2(\delta m)^{2}}}-a_{1} e^{\frac{-m}{m_{0}}}-a_{2} e^{-\frac{S}{3}}-a_{3} e^{-\tilde{\lambda}}+a_{4} e^{-\frac{\lambda}{0.3}}-a_{5} e^{-\frac{P}{0.03}}-a_{6} e^{-\frac{D}{3}} \\
-a_{7} e^{-\frac{\tilde{I}}{I_{0}}}+a_{8} e^{-\frac{I}{I_{0}}}-a_{9} e^{-\frac{X}{0.5}}+a_{10} e^{-\frac{\Delta M}{0.2 \mathrm{GeV}}}
\end{array}
$$

with $m$ the invariant mass of the candidate vertex, $S$ the vertex significance, $\lambda$ the largest angle between any tracks belonging to the vertex $(\tilde{\lambda}=1 / \lambda-0.9), P$ the vertex fit probability, $D$ the minimum normalized impact parameter, $I$ the vertex impact parameter $(\tilde{I} \times 1000+$ $24=1 /(I+0.001))$, and $X=E_{\text {vert }} / E_{\text {beam }}$ the scaled vertex energy. $\Delta M$ is the difference in the vertex mass between the pion and kaon hypotheses, and exploits the propensity for all tracks deriving from decays of the various search modes to be at high momentum. The parameters $a_{i}>0, m_{0}, I_{0}$, and $\tilde{I}_{0}$ were tuned separately for the individual search modes, while $M_{b}$ was set to $5.28 \mathrm{GeV} / \mathrm{c}^{2}$ for $B^{0}$ or $B^{+}$and $5.37 \mathrm{GeV} / \mathrm{c}^{2}$ for $B_{s}$.

For $B \rightarrow P V$ modes, the discriminator function took the form

$$
\mathcal{F}_{P V}=\left.\mathcal{F}_{\mathcal{P P}}\right|_{a_{10=0}}+a_{11} e^{-\frac{\left(m_{v}-M_{v}\right)^{2}}{2\left(\delta m_{v}\right)^{2}}}+(1-\cos (h \pi)),
$$

with $m_{v}$ the invariant mass of the vector meson candidate, and $h=\cos \theta_{h}$. The vector meson masses $M_{v}$ were set to $0.77,0.89$ and $1.02 \mathrm{GeV} / \mathrm{c}^{2}$ for $\rho, K^{* 0}$ and $\phi$ candidates, respectively, with corresponding widths $\delta m_{v}$ of $0.1,0.08$ and $0.006 \mathrm{GeV} / \mathrm{c}^{2}$, respectively.

For $B \rightarrow V V$ modes, the discriminator function took the form

$$
\mathcal{F}_{V V}=\left.\mathcal{F}_{\mathcal{P} \mathcal{P}}\right|_{a_{10=0}}+a_{11} e^{-\frac{\left(m_{v}^{(1)}-M_{v}^{(1)}\right)^{2}}{2\left(\delta m_{v}^{(1)}\right)^{2}}}+a_{12} e^{-\frac{\left(m_{v}^{(2)}-M_{v}^{(2)}\right)^{2}}{2\left(\delta m_{v}^{(2)}\right)^{2}}}+a_{13} e^{-\frac{\mathcal{M}_{V V}}{0.4 \mathrm{GeV}}}
$$

with vector meson candidates selected according to the track partition yielding vector meson masses closest to those of the search mode.

The discriminator functions were tuned for the various search modes by maximizing the separation between the SLD MC sample (which contains no charmless hadronic $B$ decays) and separately generated MC samples representing each individual search mode. The signal region for each search mode was then defined according to a cut on the output of the corresponding discriminator function. For each search mode, the value of this cut was selected in an unbiased way by minimizing the average expected MC Poisson upper limit $\mathcal{P}$ according to

$$
\mathcal{P}=\sum_{i=0}^{\infty} P(u, i) B r_{i}(\varepsilon)
$$

where $P(u, i)$ is the Poisson probability for finding $i$ background events given an expectation of $u$, and $B r_{i}(\varepsilon)$ is the $90 \%$ CL upper limit for the branching ratio if $i$ events are found. The expected signal efficiency from the MC simulation at these optimal points ranged between $24.8 \%$ and $37.9 \%$ for the various search modes, with expected backgrounds of between 0.0 and 0.48 events. The efficiency is that for all $B$ meson signal events, regardless of whether the decay occurred in the fiducial region of the detector, but does not take into account the branching ratios into fully-charged two-body final states for the vector mesons in the $B \rightarrow P V$ and $B \rightarrow V V$ modes. 
The signal efficiencies were determined from the SLD MC simulation, and thus are subject to modeling uncertainties. The efficiency of the SLD tracking system was constrained by studying the track multiplicity distributions of inclusively tagged $Z^{0} \rightarrow b \bar{b}$ events, which are identified with approximately $98 \%$ purity by the SLD [9]. The kinematic distributions of tracks from such events are well constrained by measurements of $B$ meson decay at the $\Upsilon(4 S)$ [10], as well as measurements of heavy-quark associated multiplicity at the $Z^{0}$ pole [11]. The resulting comparison of the momentum dependence of the multiplicity between inclusively tagged $\mathrm{MC}$ and data events indicated a deficit of $\sim 5 \%$ in the tracking efficiency below $1.5(0.8) \mathrm{GeV} / \mathrm{c}$ for the VXD2 (VXD3) data sample, leading to a reduction in the estimated signal mode efficiency of $\delta \varepsilon / \varepsilon \simeq 1-2 \%$.

The possibility of longitudinal polarization of the vector mesons in the $V V$ decay modes has been considered. A longitudinally polarized vector meson will decay with a $\cos ^{2} \theta_{h}$ distribution, with tracks from vector mesons decaying with small $\theta$ tending to be reconstructed less efficiently, due to the relatively low momentum of the backward-going track, as well as the resulting angular proximity of the two decay tracks. The signal MC assumes $50 \%$ longitudinal polarization for the vector mesons from $V V$ decays. Assuming a uniform probability distribution between $0 \%$ and $100 \%$ polarization, the resulting relative systematic error in the efficiency of the $V V$ decay mode reconstruction is $\Delta \varepsilon / \varepsilon=1.5 \%$.

The momentum resolution at high momentum was studied by comparing the width of the reconstructed mass peak between data and $\mathrm{MC}$ for a sample of exclusively reconstructed $D^{+} \rightarrow K^{-} \pi^{+} \pi^{+}$decays. To account for the somewhat larger width observed in data, the $\mathrm{MC}$ momentum distribution was smeared according to $1 / p_{\perp} \rightarrow 1 / p_{\perp}+$ Gaussian, for a Gaussian width of $0.002(0.001)(\mathrm{GeV} / \mathrm{c})^{-1}$ for the VXD2 (VXD3) data sample. The resulting change in the $\mathrm{MC}$ mass width, for example for the $B^{+} \rightarrow \rho^{0} \pi^{+}$search mode, is from 146 to $184 \mathrm{MeV} / \mathrm{c}^{2}$ for the VXD2 data sample. The resulting efficiency loss varied between $\delta \varepsilon / \varepsilon \simeq 2-5 \%$. Smearing of the radial and longitudinal track origin parameters, constrained by comparisons of $r-\phi$ and $r-z$ impact parameter distributions between data and MC, yielded an additional efficiency loss of $\delta \varepsilon / \varepsilon \simeq 2-4 \%$. As a cross check, after the inclusion of the above corrections in the MC efficiency calculation, the number of reconstructed $D^{+} \rightarrow K^{-} \pi^{+} \pi^{+}$decays is within $3 \%$ of the MC expectation, well within the experimental uncertainty on the $D^{+} \rightarrow K^{-} \pi^{+} \pi^{+}$branching fraction and the $D^{+}$production rate.

Application of the various search mode selection algorithms to the full 1993-8 SLD data sample yielded a total of four distinct candidate events $\left(E_{1}-E_{4}\right)$ which populated the signal regions of six separate search modes. The events observed (background expected) in each of these modes were as follows: event $E_{1}$ for $B^{0} \rightarrow \rho^{0} \rho^{0}(0.31)$; events $E_{1}, E_{2}$ for $B^{0} \rightarrow \bar{K}^{* 0} \rho^{0}$ (0.49); events $E_{1}, E_{2}, E_{3}$ for $B^{0} \rightarrow \bar{K}^{* 0} K^{* 0}(0.27)$; event $E_{4}$ for $B^{0} \rightarrow \phi \bar{K}^{* 0}(0.14)$; event $E_{1}$ for $B_{s}^{0} \rightarrow \bar{K}^{* 0} \rho^{0}(0.34)$; and events $E_{1}, E_{3}$ for $B^{0} \rightarrow \bar{K}^{* 0} K^{* 0}(0.17)$. For the remaining search modes, no events were seen.

Thirteen of fifteen MC events which passed the full selection criteria for at least one of the search modes had at least one identified track coming from a $B$ meson decay, with $B \rightarrow D \pi$ accounting for approximately one half of these. In four of the thirteen cases, reconstructed rest mass missing due to undetected charged or neutral particles was supplied by random fragmentation tracks.

Figure 1 shows the expected signal and background distributions as a function of the 
discriminator output from the MC simulation of a typical mode - the $B^{0} \rightarrow \rho^{0} \rho^{0}$ mode for the VXD-2 running period - assuming a branching fraction $\operatorname{Br}\left(B^{0} \rightarrow \rho^{0} \rho^{0}\right)=10^{-4}$. Figure 2 shows the relative rate between data and $\mathrm{MC}$ of the inclusion of background as the discriminator cut is relaxed, for the same sample. At a branching ratio of $10^{-4}$, a clear signal is expected, while backgrounds seem to be well modeled.

The mode for which the observed signal was least likely to be accounted for by a statistical fluctuation in the expected background was $B^{0} \rightarrow \bar{K}^{* 0} K^{* 0}$. The Poisson likelihood of an expected background of 0.27 events fluctuating to three or more events is $0.27 \%$, but depends strongly on the value of the expected background. A study for this mode similar to that of Fig. 2 yielded an additional 11 events, compared to a MC expectation of an additional 3 events. Thus, for this mode there is reason to believe that the background is underestimated, and so, as for other modes, only an upper limit will be quoted.

The branching ratio upper limits $L$ are related to the statistical upper limits $\alpha$ on the number of observed events according to $\alpha=S \cdot L ; S=N_{B} \cdot \varepsilon$, with $N_{B}$ the estimated number of applicable $B$ meson decays, and $\varepsilon$ the estimated efficiency for reconstructing the given signal mode. The number of $B^{+}\left(B^{0}\right)$ and $B_{s}^{0}$ meson decays in the full SLD data sample is estimated from the measured SLD sample luminosity and known $B$ meson production rates to be $(1.02 \pm 0.05) \times 10^{5}$ and $(0.27 \pm 0.05) \times 10^{5}$, respectively. It has been assumed that $(21.7 \pm 0.1) \%$ of hadronic $Z^{0}$ decays involve primary $b$ quarks, and of these, $\left(39.7_{-2.2}^{+1.8}\right) \%$ are $B^{0}$ or $B^{+}$decays, and $\left(10.5_{-2.2}^{+1.8}\right) \%$ are $B_{s}^{0}$ decays [5]. The uncertainty in the signal mode efficiencies was conservatively estimated to be the total difference in the MC efficiency estimate with and without the extra momentum, tracking efficiency, and track origin parameter smearing. Including the additional $V V$ mode polarization systematic error, as well as the MC statistical error of $\Delta \varepsilon / \varepsilon \simeq 2-5 \%$, due to the limited size of the generated signal mode samples, the total modeling error was between $6-10 \%$ for all modes.

Table 1 exhibits the number of candidate events, expected background, efficiency, sensitivity (S), and resulting 90\% CL upper limits for both the Bayesian [12 and Classical [13] approaches for the 24 search modes. Each four-prong mode limit presented here, with the exception of $B^{0} \rightarrow \phi \bar{K}^{* 0}$ and $B^{0} \rightarrow \phi \phi$, either improves upon the existing limit [5], or is the first available limit for the given mode. Two of the two-prong $B_{s}^{0}$ modes $\left(\pi^{+} \pi^{-}\right.$and $\left.K^{-} \pi^{+}\right)$ are competitive with existing limits [5]. Furthermore, a comparison of the probabilty distribution for the combination of the $\rho^{0} \pi^{+}$and $K^{* 0} \pi^{+}$modes with that implied by the DELPHI result $\operatorname{BR}\left(B^{+} \rightarrow \rho^{0} \pi^{+}, K^{* 0} \pi^{+}\right)=\left(1.7_{-0.8}^{+1.2} \pm 0.2\right) \times 10^{-4}$ yields only a $10 \%$ probability that the two measurements are consistent with the same central value.

In conclusion, the excellent tracking capabilities of the SLD detector have enabled the SLD to establish a number of unique or competitive limits on the decay of $B$ mesons to exclusive charmless final states. In particular, most of the four-prong quasi two-body limits presented here are the most stringent limits available. In addition, the SLD limits of $\operatorname{BR}\left(B^{+} \rightarrow \rho^{0} \pi^{+}\right)<0.83 \times 10^{-4}$ and $\operatorname{BR}\left(B^{+} \rightarrow K^{* 0} \pi^{+}\right)<1.19 \times 10^{-4}$ (90\% CL) rule out a DELPHI observation of the sum of these two modes [3] in favor of more stringent limits from CLEO [⿴囗十.

We thank the SLAC accelerator department for outstanding efforts on our behalf. This work was supported by the U.S. Department of Energy and National Science Foundation, the UK Particle Physics and Astronomy Research Council, the Istituto Nazionale di Fisica Nucleare of Italy and the Japan-US Cooperative Research Project on High Energy Physics. 


\section{REFERENCES}

[1] S. J. Richichi et al., CLEO-CONF 99-12, August, 1999.

[2] N. G. Deshpande and J. Trampetic, Phys. Rev. D 41. 895 (1990); L.-L. Chau et al., Phys. Rev. D 43, 2176 (1991); erratum Phys. Rev. D 58, 19902 (1998); A. Deandrea et al., Phys. Lett. B 318, 549 (1993); ibid., 320, 170 (1994); R. Fleischer, Z. Phys. C 58, 483 (1993); ibid., Phys. Lett. B 332, 419 (1994); G. Kramer, W. F. Palmer and H. Simma, Zeit. Phys. C 66, 429 (1995); D. Du and L. Guo, Z. Phys C 75, 9 (1997); D. Ebert, R. N. Faustov and V. O. Galkin, Phys. Rev. D 56, 312 (1997); N. G. Deshpande, B. Dutta and S. Oh, Phys. Rev. D 57, 5723 (1998); M. Ciuchini et al., Nucl. Phys. B 512, 3 (1998); A. Ali, G. Kramer and C.-D. Lu, Phys. Rev. D 59, 014005 (1999); Y.-H. Chen et al., hep-ph/9903453, March, 1999.

[3] W. Adam et al., Z. Phys. C 72, 207 (1996).

[4] Y. Gao et al., hep-ex/9904008, May, 1999.

[5] C. Caso et al., Eur. Phys. J. C3, 1 (1998).

[6] K. Abe et al., Phys. Rev. D53, 1023 (1996).

[7] K. Abe et al., Nucl. Instr. \& Meth. A400, 287 (1997).

[8] K. Abe et al., Nucl. Instr. \& Meth. A409, 243 (1998).

[9] David J. Jackson, Nucl. Instr. \& Meth. A388, 247 (1997).

[10] M. S. Alam et al., Phys. Rev. Lett. 49, 357 (1982).

[11] K. Abe et al., Phys. Lett. B386, 475 (1996), P. Abreu et al., Phys. Lett. B347, 447 (1995), R. Akers et al., Phys. Lett. B352, 176 (1995).

[12] R. D. Cousins et al., Nucl. Instr. \& Meth. A320, 331 (1992).

[13] G. J. Feldman et al., Phys. Rev. D57, 3873 (1998). 
${ }^{\dagger}$ The SLD Collaboration

Kenji Abe, ${ }^{(21)}$ Koya Abe, ${ }^{(33)}$ T. Abe, ${ }^{(29)}$ I. Adam, ${ }^{(29)}$ T. Akagi, ${ }^{(29)}$ H. Akimoto, ${ }^{(29)}$ N.J. Allen, ${ }^{(5)}$ W.W. Ash,${ }^{(29)}$ D. Aston, ${ }^{(29)}$ K.G. Baird, ${ }^{(17)}$ C. Baltay, ${ }^{(40)}$ H.R. Band, ${ }^{(39)}$

M.B. Barakat, ${ }^{(16)}$ O. Bardon, ${ }^{(19)}$ T.L. Barklow, ${ }^{(29)}$ G.L. Bashindzhagyan, ${ }^{(20)}$

J.M. Bauer, ${ }^{(18)}$ G. Bellodi, ${ }^{(23)}$ A.C. Benvenuti, ${ }^{(3)}$ G.M. Bilei, ${ }^{(25)}$ D. Bisello, ${ }^{(24)}$

G. Blaylock, ${ }^{(17)}$ J.R. Bogart, ${ }^{(29)}$ G.R. Bower, ${ }^{(29)}$ J.E. Brau, ${ }^{(22)}$ M. Breidenbach, ${ }^{(29)}$

W.M. Bugg, ${ }^{(32)}$ D. Burke, ${ }^{(29)}$ T.H. Burnett, ${ }^{(38)}$ P.N. Burrows, ${ }^{(23)}$ R.M. Byrne, ${ }^{(19)}$

A. Calcaterra, ${ }^{(12)}$ D. Calloway, ${ }^{(29)}$ B. Camanzi, ${ }^{(11)}$ M. Carpinelli, ${ }^{(26)}$ R. Cassell, ${ }^{(29)}$

R. Castaldi, ${ }^{(26)}$ A. Castro, ${ }^{(24)}$ M. Cavalli-Sforza, ${ }^{(35)}$ A. Chou, ${ }^{(29)}$ E. Church, ${ }^{(38)}$

H.O. Cohn, ${ }^{(32)}$ J.A. Coller, ${ }^{(6)}$ M.R. Convery, ${ }^{(29)}$ V. Cook, ${ }^{(38)}$ R.F. Cowan, ${ }^{(19)}$

D.G. Coyne, ${ }^{(35)}$ G. Crawford, ${ }^{(29)}$ C.J.S. Damerell, ${ }^{(27)}$ M.N. Danielson, ${ }^{(8)}$ M. Daoudi, ${ }^{(29)}$

N. de Groot, ${ }^{(4)}$ R. Dell'Orso, ${ }^{(25)}$ P.J. Dervan, ${ }^{(5)}$ R. de Sangro, ${ }^{(12)}$ M. Dima, ${ }^{(10)}$

D.N. Dong, ${ }^{(19)}$ M. Doser, ${ }^{(29)}$ R. Dubois, ${ }^{(29)}$ B.I. Eisenstein, ${ }^{(13)}$ I.Erofeeva, ${ }^{(20)}$

V. Eschenburg, ${ }^{(18)}$ E. Etzion, ${ }^{\left({ }^{(3)}\right.}$ S. Fahey, ${ }^{(8)}$ D. Falciai, ${ }^{(12)}$ C. Fan, ${ }^{(8)}$ J.P. Fernandez, ${ }^{(35)}$

M.J. Fero, ${ }^{(19)}$ K. Flood, ${ }^{(17)}$ R. Frey, ${ }^{(22)}$ J. Gifford, ${ }^{(36)}$ T. Gillman, ${ }^{(27)}$ G. Gladding, ${ }^{(13)}$

S. Gonzalez, ${ }^{(19)}$ E.R. Goodman, ${ }^{(8)}$ E.L. Hart, ${ }^{(32)}$ J.L. Harton, ${ }^{(10)}$ K. Hasuko, ${ }^{(33)}$

S.J. Hedges, ${ }^{(6)}$ S.S. Hertzbach, ${ }^{(17)}$ M.D. Hildreth, ${ }^{(29)}$ J. Huber, ${ }^{(22)}$ M.E. Huffer, ${ }^{(29)}$

E.W. Hughes, ${ }^{(29)}$ X. Huynh, ${ }^{(29)}$ H. Hwang, ${ }^{(22)}$ M. Iwasaki, ${ }^{(22)}$ D.J. Jackson, ${ }^{(27)}$

P. Jacques, ${ }^{(28)}$ J.A. Jaros, ${ }^{(29)}$ Z.Y. Jiang, ${ }^{(29)}$ A.S. Johnson, ${ }^{(29)}$ J.R. Johnson, ${ }^{(39)}$

R.A. Johnson, ${ }^{(7)}$ T. Junk, ${ }^{(29)}$ R. Kajikawa, ${ }^{(21)}$ M. Kalelkar, ${ }^{(28)}$ Y. Kamyshkov, ${ }^{(32)}$

H.J. Kang, ${ }^{(28)}$ I. Karliner, ${ }^{(13)}$ H. Kawahara, ${ }^{(29)}$ Y.D. Kim, ${ }^{(30)}$ M.E. King, ${ }^{(29)}$ R. King, ${ }^{(29)}$

R.R. Kofler, ${ }^{(17)}$ N.M. Krishna, ${ }^{(8)}$ R.S. Kroeger, ${ }^{(18)}$ M. Langston, ${ }^{(22)}$ A. Lath, ${ }^{(19)}$

D.W.G. Leith, ${ }^{(29)}$ V. Lia, ${ }^{(19)}$ C.Lin, ${ }^{(17)}$ M.X. Liu, ${ }^{(40)}$ X. Liu, ${ }^{(35)}$ M. Loreti, ${ }^{(24)}$ A. Lu, ${ }^{(34)}$

H.L. Lynch, ${ }^{(29)}$ J. Ma, ${ }^{(38)}$ M. Mahjouri, ${ }^{(19)}$ G. Mancinelli, ${ }^{(28)}$ S. Manly, ${ }^{(40)}$

G. Mantovani, ${ }^{(25)}$ T.W. Markiewicz, ${ }^{(29)}$ T. Maruyama, ${ }^{(29)}$ H. Masuda, ${ }^{(29)}$ E. Mazzucato, ${ }^{(11)}$

A.K. McKemey, ${ }^{(5)}$ B.T. Meadows, ${ }^{(7)}$ G. Menegatti, ${ }^{(11)}$ R. Messner, ${ }^{(29)}$ P.M. Mockett, ${ }^{(38)}$

K.C. Moffeit, ${ }^{(29)}$ T.B. Moore, ${ }^{(40)}$ M.Morii, ${ }^{(29)}$ D. Muller, ${ }^{(29)}$ V. Murzin, ${ }^{(20)}$ T. Nagamine, ${ }^{(33)}$

S. Narita, ${ }^{(33)}$ U. Nauenberg, ${ }^{(8)}$ H. Neal, ${ }^{(29)}$ M. Nussbaum,,${ }^{(7)}$ N. Oishi, ${ }^{(21)}$

D. Onoprienko, ${ }^{(32)}$ L.S. Osborne, ${ }^{(19)}$ R.S. Panvini, ${ }^{(37)}$ C.H. Park, ${ }^{(31)}$ T.J. Pavel, ${ }^{(29)}$

I. Peruzzi, ${ }^{(12)}$ M. Piccolo, ${ }^{(12)}$ L. Piemontese, ${ }^{(11)}$ K.T. Pitts, ${ }^{(22)}$ R.J. Plano, ${ }^{(28)}$

R. Prepost, ${ }^{(39)}$ C.Y. Prescott, ${ }^{(29)}$ G.D. Punkar, ${ }^{(29)}$ J. Quigley, ${ }^{(19)}$ B.N. Ratcliff, ${ }^{(29)}$

T.W. Reeves, ${ }^{(37)}$ J. Reidy, ${ }^{(18)}$ P.L. Reinertsen, ${ }^{(35)}$ P.E. Rensing, ${ }^{(29)}$ L.S. Rochester, ${ }^{(29)}$

P.C. Rowson, ${ }^{(9)}$ J.J. Russell, ${ }^{(29)}$ O.H. Saxton, ${ }^{(29)}$ T. Schalk, ${ }^{(35)}$ R.H. Schindler, ${ }^{(29)}$

B.A. Schumm, ${ }^{(35)}$ J. Schwiening, ${ }^{(29)}$ S. Sen, ${ }^{(40)}$ V.V. Serbo, ${ }^{(29)}$ M.H. Shaevitz, ${ }^{(9)}$

J.T. Shank, ${ }^{(6)}$ G. Shapiro, ${ }^{(15)}$ D.J. Sherden, ${ }^{(29)}$ K.D. Shmakov, ${ }^{(32)}$ C. Simopoulos, ${ }^{(29)}$

N.B. Sinev, ${ }^{(22)}$ S.R. Smith, ${ }^{(29)}$ M.B. Smy, ${ }^{(10)}$ J.A. Snyder, ${ }^{(40)}$ H. Staengle, ${ }^{(10)}$ A. Stahl, ${ }^{(29)}$

P. Stamer, ${ }^{(28)}$ H. Steiner, ${ }^{(15)}$ R. Steiner, ${ }^{(1)}$ M.G. Strauss, ${ }^{(17)}$ D. Su, ${ }^{(29)}$ F. Suekane, ${ }^{(33)}$

A. Sugiyama, ${ }^{(21)}$ S. Suzuki, ${ }^{(21)}$ M. Swartz, ${ }^{(14)}$ A. Szumilo, ${ }^{(38)}$ T. Takahashi, ${ }^{(29)}$

F.E. Taylor, ${ }^{(19)}$ J. Thom, ${ }^{(29)}$ E. Torrence, ${ }^{(19)}$ N.K. Toumbas, ${ }^{(29)}$ T. Usher, ${ }^{(29)}$

C. Vannini, ${ }^{(26)}$ J. Va'vra, ${ }^{(29)}$ E. Vella, ${ }^{(29)}$ J.P. Venuti, ${ }^{(37)}$ R. Verdier, ${ }^{(19)}$ P.G. Verdini, ${ }^{(26)}$

D.L. Wagner, ${ }^{(8)}$ S.R. Wagner, ${ }^{(29)}$ A.P. Waite, ${ }^{(29)}$ S. Walston, ${ }^{(22)}$ S.J. Watts, ${ }^{(5)}$

A.W. Weidemann, ${ }^{(32)}$ E. R. Weiss, ${ }^{(38)}$ J.S. Whitaker, ${ }^{(6)}$ S.L. White, ${ }^{(32)}$ F.J. Wickens, ${ }^{(27)}$

B. Williams, ${ }^{(8)}$ D.C. Williams, ${ }^{(19)}$ S.H. Williams, ${ }^{(29)}$ S. Willocq, ${ }^{(17)}$ R.J. Wilson, ${ }^{(10)}$

W.J. Wisniewski, ${ }^{(29)}$ J. L. Wittlin, ${ }^{(17)}$ M. Woods, ${ }^{(29)}$ G.B. Word, ${ }^{(37)}$ T.R. Wright, ${ }^{(39)}$

J. Wyss, ${ }^{(24)}$ R.K. Yamamoto, ${ }^{(19)}$ J.M. Yamartino, ${ }^{(19)}$ X. Yang, ${ }^{(22)}$ J. Yashima, ${ }^{(33)}$

S.J. Yellin, ${ }^{(34)}$ C.C. Young, ${ }^{(29)}$ H. Yuta, ${ }^{(2)}$ G. Zapalac, ${ }^{(39)}$ R.W. Zdarko, ${ }^{(29)}$ J. Zhou. ${ }^{(22)}$

\section{(The SLD Collaboration)}


(1) Adelphi University, Garden City, New York 11530,

(2) Aomori University, Aomori , 030 Japan,

${ }^{(3)}$ INFN Sezione di Bologna, I-40126, Bologna, Italy,

(4) University of Bristol, Bristol, U.K.,

${ }^{(5)}$ Brunel University, Uxbridge, Middlesex, UB8 3PH United Kingdom,

(6) Boston University, Boston, Massachusetts 02215,

(7) University of Cincinnati, Cincinnati, Ohio 45221,

(8) University of Colorado, Boulder, Colorado 80309,

(9) Columbia University, New York, New York 10533,

(10) Colorado State University, Ft. Collins, Colorado 80523,

(11) INFN Sezione di Ferrara and Universita di Ferrara, I-44100 Ferrara, Italy,

${ }^{(12)}$ INFN Lab. Nazionali di Frascati, I-00044 Frascati, Italy,

(13) University of Illinois, Urbana, Illinois 61801,

(14) Johns Hopkins University, Baltimore, Maryland 21218-2686,

${ }^{(15)}$ Lawrence Berkeley Laboratory, University of California, Berkeley, California 94720,

${ }^{(16)}$ Louisiana Technical University, Ruston, Louisiana 71272,

(17) University of Massachusetts, Amherst, Massachusetts 01003,

(18) University of Mississippi, University, Mississippi 3867\%,

(19) Massachusetts Institute of Technology, Cambridge, Massachusetts 02139,

${ }^{(20)}$ Institute of Nuclear Physics, Moscow State University, 119899, Moscow Russia,

${ }^{(21)}$ Nagoya University, Chikusa-ku, Nagoya, 464 Japan,

${ }^{(22)}$ University of Oregon, Eugene, Oregon 97403,

(23) Oxford University, Oxford, OX1 3RH, United Kingdom,

(24) INFN Sezione di Padova and Universita di Padova I-35100, Padova, Italy,

${ }^{(25)}$ INFN Sezione di Perugia and Universita di Perugia, I-06100 Perugia, Italy,

${ }^{(26)}$ INFN Sezione di Pisa and Universita di Pisa, I-56010 Pisa, Italy,

${ }^{(27)}$ Rutherford Appleton Laboratory, Chilton, Didcot, Oxon OX11 0QX United Kingdom,

${ }^{(28)}$ Rutgers University, Piscataway, New Jersey 08855,

(29) Stanford Linear Accelerator Center, Stanford University, Stanford, California 94309,

${ }^{(30)}$ Sogang University, Seoul, Korea,

(31) Soongsil University, Seoul, Korea 156-743,

${ }^{(32)}$ University of Tennessee, Knoxville, Tennessee 37996,

(33) Tohoku University, Sendai 980, Japan,

(34) University of California at Santa Barbara, Santa Barbara, California 93106,

(35) University of California at Santa Cruz, Santa Cruz, California 95064,

(36) University of Victoria, Victoria, British Columbia, Canada V8W 3P6,

(37) Vanderbilt University, Nashville, Tennessee 37235,

(38) University of Washington, Seattle, Washington 98105,

(39) University of Wisconsin, Madison, Wisconsin 53706,

(40) Yale University, New Haven, Connecticut 06511. 


\section{TABLES}

\begin{tabular}{lcccccc}
\hline \hline Mode & $\varepsilon$ & $\mathrm{S}\left(\times 10^{-4}\right)$ & Bckd & Data & UL $\left(\mathrm{B} ; \times 10^{4}\right)$ & UL $\left(\mathrm{C} ; \times 10^{4}\right)$ \\
\hline$B^{0} \rightarrow \pi^{+} \pi^{-}$ & .338 & $3.46 \pm .31$ & 0.03 & 0 & 0.69 & 0.67 \\
$B^{0} \rightarrow K^{-} \pi^{+}$ & .345 & $3.53 \pm .32$ & 0.14 & 0 & 0.67 & 0.66 \\
$B^{0} \rightarrow K^{+} K^{-}$ & .341 & $3.49 \pm .28$ & 0.14 & 0 & 0.67 & 0.66 \\
\hline$B_{s}^{0} \rightarrow \pi^{+} \pi^{-}$ & .379 & $1.02 \pm .16$ & 0.03 & 0 & 2.35 & 2.32 \\
$B_{s}^{0} \rightarrow K^{-} \pi^{+}$ & .335 & $0.91 \pm .15$ & 0.10 & 0 & 2.62 & 2.61 \\
$B_{s}^{0} \rightarrow K^{+} K^{-}$ & .311 & $0.84 \pm .14$ & 0.20 & 0 & 2.77 & 2.83 \\
\hline$B^{+} \rightarrow \rho^{0} \pi^{+}$ & .272 & $2.78 \pm .26$ & 0.34 & 0 & 0.81 & 0.83 \\
$B^{+} \rightarrow \rho^{0} K^{+}$ & .264 & $2.70 \pm .24$ & 0.41 & 0 & 0.83 & 0.86 \\
$B^{+} \rightarrow K^{* 0} \pi^{+}$ & .285 & $1.94 \pm .17$ & 0.17 & 0 & 1.21 & 1.19 \\
$B^{+} \rightarrow K^{* 0} K^{+}$ & .248 & $1.69 \pm .18$ & 0.17 & 0 & 1.39 & 1.38 \\
$B^{+} \rightarrow \phi \pi^{+}$ & .301 & $1.51 \pm .12$ & 0.07 & 0 & 1.59 & 1.53 \\
$B^{+} \rightarrow \phi K^{+}$ & .321 & $1.61 \pm .12$ & 0.14 & 0 & 1.47 & 1.44 \\
\hline$B^{0} \rightarrow \rho^{0} \rho^{0}$ & .263 & $2.76 \pm .24$ & 0.31 & 1 & 1.57 & 2.86 \\
$B^{0} \rightarrow \bar{K}^{* 0} \rho^{0}$ & .253 & $1.76 \pm .15$ & 0.49 & 2 & 3.30 & 4.69 \\
$B^{0} \rightarrow \bar{K}^{* 0} K^{* 0}$ & .304 & $1.42 \pm .12$ & 0.27 & 3 & 5.27 & 1.56 \\
$B^{0} \rightarrow \phi \rho^{0}$ & .298 & $1.53 \pm .13$ & 0.14 & 0 & 1.58 & 3.84 \\
$B^{0} \rightarrow \phi \bar{K}^{* 0}$ & .295 & $1.01 \pm .08$ & 0.14 & 1 & 4.34 & 3.21 \\
$B^{0} \rightarrow \phi \phi$ & .393 & $0.74 \pm .05$ & 0.00 & 0 & 3.37 & 3.20 \\
\hline$B_{s}^{0} \rightarrow \rho^{0} \rho^{0}$ & .277 & $0.77 \pm .13$ & 0.27 & 0 & 3.06 & 7.67 \\
$B_{s}^{0} \rightarrow \bar{K}^{* 0} \rho^{0}$ & .272 & $0.50 \pm .09$ & 0.34 & 1 & 8.52 & 16.81 \\
$B_{s}^{0} \rightarrow \bar{K}^{* 0} K^{* 0}$ & .265 & $0.33 \pm .05$ & 0.17 & 2 & 18.21 & 6.17 \\
$B_{s}^{0} \rightarrow \phi \rho^{0}$ & .290 & $0.39 \pm .06$ & 0.07 & 0 & 10.02 & 11.83 \\
$B_{s}^{0} \rightarrow \phi K^{* 0}$ & .265 & $0.24 \pm .04$ & 0.14 & 0 & 12.11 & \\
$B_{s}^{0} \rightarrow \phi \phi$ & .308 & $0.21 \pm .03$ & 0.00 & 0 & 0.13 \\
\hline \hline
\end{tabular}

TABLE I. Summary of efficiency $(\varepsilon)$, sensitivity (S), expected background, number of events in the signal region, Classical (C) and Bayesian (B) 90\% CL Upper Limit for the 24 search modes. Note that the sensitivities (but not the efficiencies) take account of the branching fraction for $\phi$ or $K^{* 0}$ into a fully-charged two body final state, where applicable. 


\section{FIGURES}

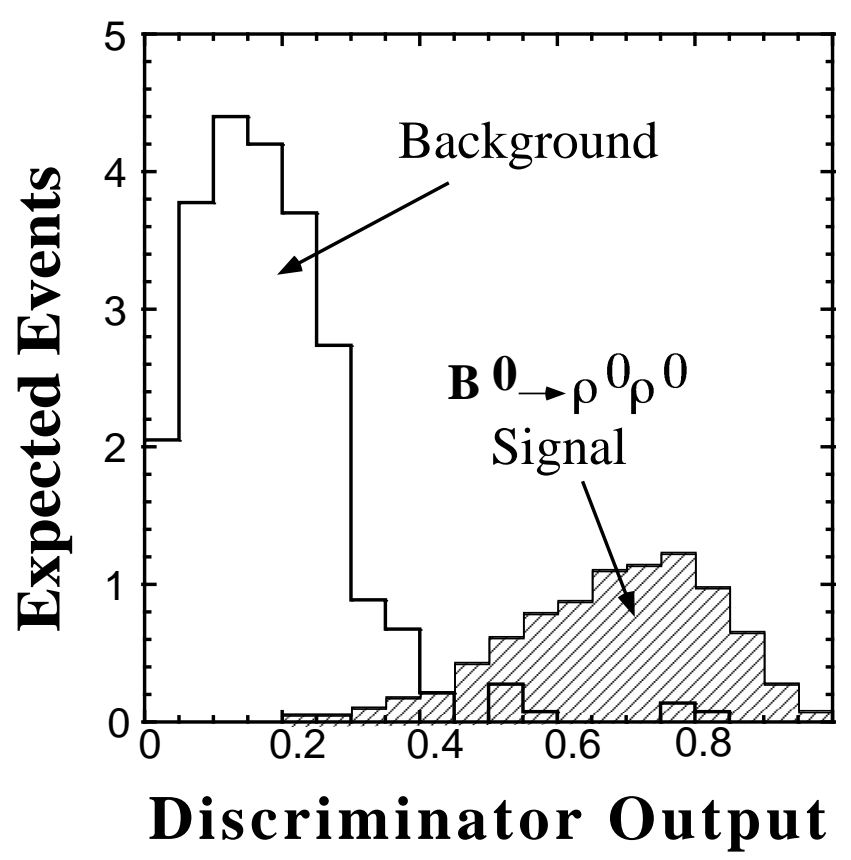

FIG. 1. Expected signal (shaded) and background for the $B^{0} \rightarrow \rho^{0} \rho^{0}$ channel, as a function of the discriminator output. The plot shown is from the VXD-3 period Monte Carlo, assuming a branching fraction $\operatorname{Br}\left(B^{0} \rightarrow \rho^{0} \rho^{0}\right)=10^{-4}$.

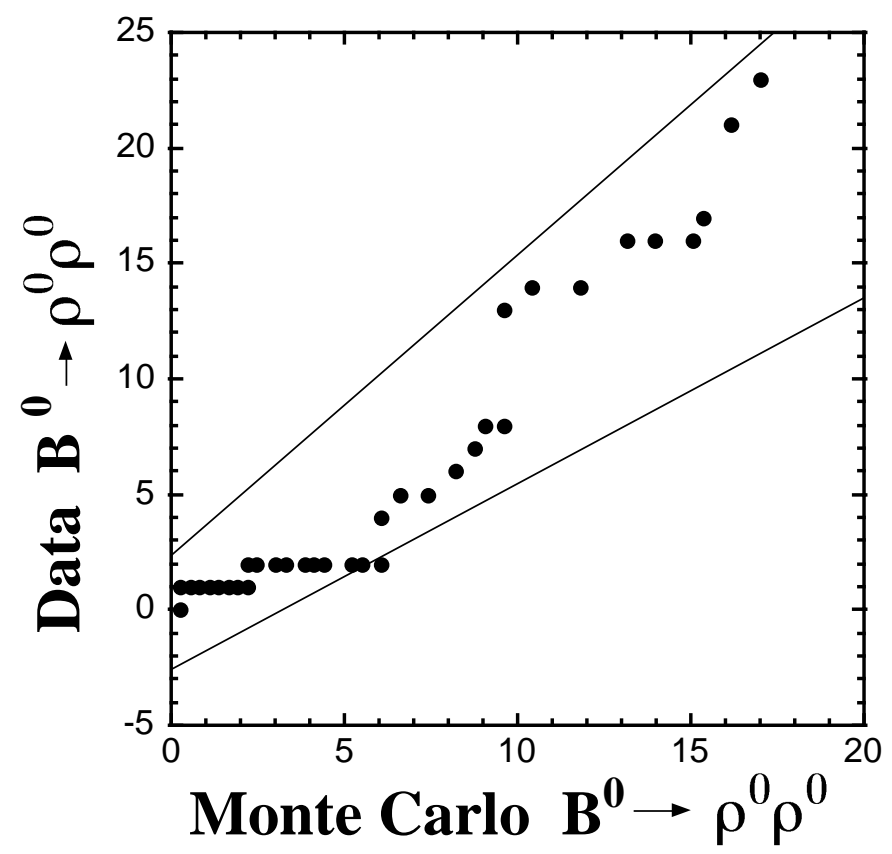

FIG. 2. Comparison between VXD-3 data and Monte Carlo of the rate of introduction of background into the $B^{0} \rightarrow \rho^{0} \rho^{0}$ sample as the discriminator function cut is relaxed. The lines represent the upper and lower $90 \%$ CL limits under the assumption that the Monte Carlo accurately models the background. 